\title{
Diabetes and data in many forms
}

\author{
G. R. Sridhar ${ }^{1}$
}

Published online: 8 December 2016

(C) Research Society for Study of Diabetes in India 2016

Traditionally, capture, analysis, and usage of data were difficult. Information technology has altered the equation between capture and usability: generation of data exploded; constraints exist in analysis and appropriate use.

The rich source of clinical and biochemical data in diabetes was usually recorded on paper. It was efficient to document, but laborious to retrieve and analyze. Availability of information technology revolutionized all these. The use of electronic medical records (EMR) makes it possible to capture biographical, clinical, and biochemical data at one point and on followup $[1,2]$.

In its early years, biological science was descriptive: observing and classifying different parts of the biological system. The Human Genome Project provided a path for the ultimate in a reductionist approach: defining the units of information in terms of the nucleotide sequences which code for biological effects [3]. It is now widely accepted that information science and molecular biology have shared concepts.

To bring information technology into clinical context is beset with difficulties. Primarily, it depends on the purpose for which the data would be used (clinical care, clinical focus on a narrow area, clinical audit - although these are not mutually exclusive). Ultimately, there is a tradeoff between the depth of information that is gathered and the time that is available for each subject. This is a crucial aspect in converting paper-based record system to electronic medical records, as

Based on the Presidential oration at the Endocrine Society of India Annual Conference held at PGIMER Chandigarh on 15 November 2014

G. R. Sridhar

sridharvizag@gmail.com

1 Endocrine and Diabetes Centre, 15-12-15 Krishnanagar, Visakhapatnam 530002, India the advantages of the latter are many. Good software is given, but the bottleneck in converting to EMR is "process design, understanding and supporting workflows, and the economic and social aspects of organizational change."

Once the system is in place, advantages are obvious: structured entry of information, near-instantaneous recall, and built in rules for diagnosis and advice $[1,2]$.

The data can be utilized in ways other than for the purpose it was originally obtained. For instance, it could be subjected to predictive modeling using neural network methods. In the current issue, Duggal et al. used machine learning methods to predict readmission of patients who were discharged from a hospital [4]. From 9381 records, random forest was the optimal classifier using area under precision-recall curve to identify risk factors. Neural network methods have the ability to "learn" as larger sets of data are provided. It differs from statistical methods, which operate on the available circumscribed data. Machine-learning tools were used in other applications such as detecting adverse drug events from electronic medical records [5].

Access to mobile telephony led to development of mHealth apps. In this issue of the journal, Jha et al. [6] compared the effectiveness of electronic health and mobile health platform with conventional care in diabetes. A pilot study from a tertiary care hospital showed a reduction in HbAlc. Diabetes knowledge and quality of life were better in the group managed by electronic health and mobile health record.

The World Health Organization published a document on mHealth strategy [7]. eHealth is defined as the "use of information and communication technology for health" with an objective to improve service delivery and outcomes utilizing computers, internet, and mobile phones. Advantages eHealth include promotion of healthy lifestyle and improved decisions by ensuring access to care even in remote places. Of relevance to diabetes, eHealth and mHealth can be employed in remote 
monitoring, health education, and continual training in selfcare. Studies from India have shown mHealth is feasible and improves health outcomes in subjects with diabetes. Text messaging improved health behavior related to diabetes [8]. Similarly, a pilot study of mHealth intervention was compared to usual care in three metropolitan cities across India over a 6month period [9]. The Gather system, built on behavioral change theories to support self-management and to improve communication with physicians, resulted in improved A1c levels, similar to an earlier study reported by Shetty et al. [10]. A systematic review on web-based remote monitoring systems in the self-management of type 2 diabetes concluded that there is complexity involved in the technology as well as in its implementation [11]. These must be addressed.

Rapid advances in technology are bringing in innovative methods of low-cost, efficient care for subjects with diabetes [12]. These have been described as affording "a more fluid, real-time, and patient-centric" treatment by way of access to education material, integration of biochemical data in improving self-care [13]. More than 1000 mobile apps exist, related to chronic diseases including diabetes. The apps related to diabetes allow logging and tracking patient data, as well as exporting the data to EMRs. A number of publications show that these apps are feasible and effective in improving clinical outcomes [14]. Work in progress consists of being able to add notes to data, tracking lab results, and employing identifiers such as bar codes [15]. These apps are expected to be certified for quality control by regulatory agencies.

There have been interesting attempts where data is analyzed to learn how built environment is related to health and well-being. Built environment refers to environments that are modified by humans, which influences their behavior leading to changes in lifestyle [16]. Urban sprawl typically found in developed western countries contributes to increasing sedentary lifestyle and obesity and diabetes.

Psychosocial measures were assessed in relation to built environment using Guttman's smallest space analysis [17]. Relations among the psychosocial measures were accounted for by "one facet with three axial sets of variables:" (a) positive well-being and energy; (b) satisfaction, impact, and social worry and diabetes worry; and (c) anxiety and depression. Prevention methods can be devised keeping the relation of built environment and psychosocial stressors. It is of interest that the data was obtained over the course of years primarily for evaluation of various psychosocial aspects of diabetes [18, 19].

The data can be analyzed using neural network methods to predict the psychological outcome based on key clinical and biographical factors [20]. Similarly, clinical data can be analyzed to identify trends in the prevalence and other characters of diseases [21, 22].

But it is really after the publication of the human genome, in all its iterations, that biological data and information science were recognized to be intertwined. While in the past, data is difficult to obtain and analyze, newer molecular biological approaches turned the cart around-where a lot more data were available. Challenges exist to develop statistical, mathematical, and computational methods to understand and utilize [23].

There has been continuing documentation of gene expression studies in different situations: among offspring who had one parent with type 2 diabetes [24], PPARG (Pro12Ala) polymorphisms [25], and variants of a TCF7L2 gene in the north east part of Uttar Pradesh [26]. An earlier editorial in the journal summarized aspects of such studies [27].

The avowed practical implementation of the HGP was personalization of drugs; although benefits have been hard to come by, attempts are being made to match genetic profile with the individual. A review of personalized drug use in diabetes is published in this issue [28]. Although genetic variants and metabolomics studies using omics technologies have the potential to be used as biomarkers [29], translatable results are scarce. Despite a number of common variants being associated with type 2 diabetes, they explain only a very small fraction of the disease heritability. A recent effort to improve the predictive value by studying infrequent and rare variants showed they did not have a significant role in predisposing to the disease [30]. Resultantly, the major limitation in applying diabetes genomics to clinical care is the lack of genomic findings which can be used in clinical practice [31]. The way forward seems to consist of improved understanding of genetic variant interactions with environmental factors [32]. Given the low penetrance of most alleles in type 2 diabetes and poor predictive value, methods must be devised to communicate the risks accruing from genetic factors so that beneficial health outcomes can be achieved [33]. Alongside, there is an increasing need for trained researchers at the "intersection of computer science, statistics, mathematics, and their discipline of interest" to deal with data-rich but (as yet) discovery-poor situation [34, 35].

Of the many ways in which genomic data can be analyzed, the ability to impute evolutionary pathways, as demonstrated by the phylogenetic and promoter analysis of IAP gene with diabetes in the current issue of the journal, is exciting [36]. The phylogenetic and the gene promoter analysis suggested that "regulatory elements for beta cell death caused by pancreatic amyloidosis" could lead to T2DM. This is similar to the hypothesis based on bioinformatics analysis that the enzyme butyrylcholinesterase could be related to the pathogenesis of Alzheimer's disease and type 2 diabetes mellitus [37]. The hypothesis was followed up by an animal study in which streptozotocin-induced diabetes in albino Wistar rats showed a decline in cognitive function and an increased serum butyrylcholinesterase [38]. A series of other studies have suggested that it could be one of the mediators between type 2 diabetes mellitus and Alzheimer's disease [39]. Of greater interest is 
the potential of evaluating individuals having variant butyrylcholinesterase proteins and their susceptibility or protection from metabolic syndrome and its associated abnormalities $[40,41]$.

Advances in these fields not only require conceptual and technical collaboration to devise treatable options [35] but could also involve ethical issues such as the ownership of data and providing access of genetic data to the participants, i.e., in other words, to answer the question "who owns the data [42]?"

Besides analysis of biological data, availability of other forms of data provides innovative methods to assess poverty and to understand how social interactions are related to health outcomes [43, 44].

So we are at a juncture where there is a deluge of data. Methods must be devised to collect, annotate, and understand it, rather than be overwhelmed. One can expect to deal not just with science but with society, ethics, and law. Interesting times await.

\section{References}

1. Sridhar GR, Appa Rao A, Muraleedharan MV, Jaya Kumar RV, Yarabati V. Electronic medical records and hospital management systems for management of diabetes. Diabetes \& Metabolic Syndrome: Clinical Research \& Reviews. 2009;3:55-9.

2. Sridhar GR, Murali G. Computerization of data in diabetes centers. Int J Diabetes Dev Ctries. 2011;31:48-50.

3. Pranavchand R, Reddy BM. Genomics era and complex disorders: implications of GWAS with special reference to coronary artery disease, type 2 diabetes mellitus, and cancers. J Postgrad Med. 2016;62:188-98.

4. Duggal R, Shukla S, Chandra S, et al. Predictive risk modelling for early hospital readmission of patients with diabetes in India. Int $\mathbf{J}$ Diabetes Dev Ctries. 2016; doi:10.1007/s13410-016-0511-8.

5. Zhao J, Henriksson A, Asker L, Bostrom A. Predictive modeling of structured electronic health records for adverse drug event detection. BMC Medical Informatics and Decision Making. 2015;15(Suppl 4):51.

6. Jha S, Dogra S, Yadav A, et al. A prospective observational study to assess the effectiveness of an electronic health (E-health) and mobile health (M-health) platform versus conventional care for the management of diabetes mellitus. Int J Diabetes Dev Ctries. 2016; doi:10.1007/s13410-016-0501-x.

7. WHO. Towards the development of an mHealth strategy: a literature review. The Millennium Villages Project. 2008.

8. Pfammatter A, Spring B, Saligram N, et al. mHealth intervention to improve diabetes risk behaviors in India: a prospective, parallel group cohort study. J Med Internet Res. 2016;18:e207.

9. Kleinman NL, Shah A, Shah S, Phatak S, Viswanathan V. Impact of the Gather mHealth system on A1c: primary results of a multisite randomized clinical trial among people with type 2 diabetes in India. Diabetes Care. 2016;39:e169-70.

10. Shetty AS, Chamukuttan S, Nanditha A, Raj RKC, Ramachandran A. Reinforcement of adherence to prescription recommendations in Asian Indian diabetes patients using short message service (SMS) - a pilot study. J Assoc Physicians India. 2011;59:711-4.
11. Muschcab H, Kernohan WG, Wallace J, Martin S. Web-based remote monitoring systems for self-managing type 2 diabetes: a systematic review. Diabetes Technol Ther. 2015;17:498-509.

12. Spearson CL, Mistry A. Several aspects of internet and web-based technology in diabetes management. Diabetes Spectrum. 2016;29: 245-8.

13. Davis B, Pan E, Walker J, et al. Benefits of information technologyenabled diabetes management. Diabetes Care. 2007;30:1137-42.

14. Hou C, Carter B, Hewitt J, Francisa T, Mayor S. Do mobile phone applications improve glycemic control $\left(\mathrm{HBA}_{1 \mathrm{c}}\right)$ in the selfmanagement of diabetes? A systemic review, meta-analysis, and GRADE of 14 randomized trials. Diab Care. 2016;39:2089-95.

15. Neuman B, Stefanik M, Gonzalvo J, Weter Z. Diabetes mHealth applications: where are we now? AADE in Practice 2016; 28-32.

16. Pasala SK, Appa Rao A, Sridhar GR. Built environment and diabetes. Intl J Diab Dev Cntr. 2010;30:63-8.

17. Sridhar GR, Sudhir Kumar P, Venkata P, et al. Built environment factors, psychosocial factors and diabetes mellitus: a south Indian study. Indian J Clin Med. 2010;1:15-33.

18. Sridhar GR, Madhu K. Psychosocial and cultural issues in diabetes mellitus. Curr Sci. 2002;83:1556-64.

19. Sridhar GR, Madhu K, Veena S, Madhavi R, Sangeetha BS, Rani A. Living with diabetes: Indian experience. Diabetes \& Metabolic Syndrome: Clinical Research \& Reviews. 2007;1:181-7.

20. Narasinga Rao MR, Sridhar GR, Madhu K, Appa RA. A clinical decision support system using multi-layer perceptron neural network to predict quality of life in diabetes. Diab Metab Syndr: Clin Res Rev. 2010;4:57-9.

21. Sridhar GR. Diabetes in India: snapshot of a panorama. Curr Sci. 2002;83:791.

22. Sridhar GR, Putcha V, Lakshmi G. Time trends in the prevalence of diabetes mellitus :ten year analysis from southern India (19942004) on 19,072 subjects with diabetes. J Assoc Physicians India. 2010;58:290-4.

23. Sridhar GR. The emerging use of genomics and proteomics in endocrinology. Intl J Diab Dev Countries. 2002;22:135-8.

24. Safi SZ, Qvist R, Chinna K, et al. Gene expression profiling of the peripheral blood mononuclear cells of offspring of one type 2 diabetic parent. Int J Diabetes Dev Ctries. 2015; doi:10.1007/s13410015-0369-1.

25. Paramasivam D, Safi SZ, Qvist R, et al. Role of PPARG (Pro12Ala) in Malaysian type 2 diabetes mellitus patients. Int J Diabetes Dev Ctries. 2016; doi:10.1007/s13410-015-0462-5.

26. Yadav SK, Rashmi, Tripathi KK, et al. Association of TCF7L2 gene variant with T2DM, T1DM and gestational diabetes in the population of northeastern UP. India Int J Diabetes Dev Ctries. 2016; doi:10.1007/s13410-016-0490-9.

27. Sridhar GR, Ravindranath D, Sandosh P. Emerging face of genetics, genomics and diabetes. Intl J Diab Dev Countries. 2013;33:183-5.

28. Boriboonhirunsarn D, Tangthasana S. Effects of pre-pregnancy weight on incidence of large for gestational age newborn in pregnant women with gestational diabetes mellitus. Int J Diabetes Dev Ctries. 2015; doi:10.1007/s13410-015-0381-5.

29. Pearson ER. Personalized medicine in diabetes: the role of 'omics' and biomarkers. Diabet Med. 2016;33:712-7.

30. Fuschsberger $\mathrm{C}$ et al. The genetic architecture of type 2 diabetes. Nature. 2016;536:41-7.

31. Floyd JS, Psaty BM. The application of genomics in diabetes: barriers to discovery and implementation. Diab Care. 2016;39:185869.

32. Tallapragada DSP, Bhaskar S, Chandak GR. New insights from monogenic diabetes for "common" type 2 diabetes. Front Genet. 2015;6:251. doi:10.3389/fgene.2015.00251.

33. Ozdemir V, Burke W, Khoury MJ, Knoppers BM, Zimmern R. Genomics and public health. In: Detals R, Gulliford M, Karim 
QA, Tan CC, editors. Oxford textbook of global public health. New York: Oxford Univ Press; 2015. p. p140-61.

34. Chandler V. Turning data into discovery. The Scientist June 2015; http://www.the-scientist.com/?articles. view/articleNo/43021/title/Turning-Data-into-Discovery/, ).

35. Sridhar GR, Lakshmi G. Bioinformatics, genomics and diabetes. In: Lakshmi PV, Zhou W, Satheesh P, editors. Computational intelligence techniques in health care. Singapore: Springer; 2016. p. 1-18.

36. Singh V, Saluja N. Phylogenetic and promoter analysis of islet amyloid polypeptide gene causing type 2 diabetes in mammalian species. Int J Diabetes Dev Ctries. 2016; doi:10.1007/s13410-0160508-3.

37. Sridhar GR, Hanuman T, Rao AA, et al. Alzheimer's disease and type 2 diabetes mellitus: the cholinesterase connection? Lipids Health Dis. 2006;5:28. doi:10.1186/1476-511X-5-28.

38. Rao AA, Siva Reddy C, Sridhar GR. Enhanced butyrylcholinesterase activity may be the common link in triggering low-grade systemic inflammation and decrease in cognitive function in diabetes mellitus and Alzheimer's disease. Curr Nutr Food Sci. 2008;4: 213-6.

39. Sridhar GR, Lakshmi G, Nagamani G. Emerging links between type 2 diabetes and Alzheimer's disease. World J Diabetes. 2015;6:744-51.

40. Alkuraya FS. Natural human knockouts and the era of genotype to phenotype. Genome Medicine. 2015;7:48.

41. Sridhar GR, Rao AA, Srinivas K, et al. Butyrylcholinesterase in metabolic syndrome. Med Hypotheses. 2010;75:648-51.

42. Nelson S. Geneticists should offer data to participants. Nature. 2016;539:7.

43. Blumenstock JE. Fighting poverty with data. Science. 2016;353: 753.

44. Hobbs WR, Burke M, Christakis NA, Fowler JH. Online social integration is associated with reduced mortality risk. PNAS. 2016;113:12980-4. 